\title{
Not out of the woods yet: signatures of the prolonged negative genetic consequences of a population bottleneck in a rapidly re-expanding wader, the black-faced spoonbill Platalea minor
}

\author{
Shou-Hsien $\mathrm{Li}^{1}$, Yang Liu ${ }^{2}$, Chia-Fen Yeh ${ }^{1}$, Yuchen Fu ${ }^{3}$, Carol K. L. Yeung ${ }^{3}$, Chun-cheng \\ $\mathrm{Lee}^{3}$, Chi-Cheng Chiu ${ }^{1}$, Tung Hui Kuo ${ }^{3}$, FT Chan ${ }^{4}$, Yu-Chia Chen ${ }^{3}$, Wen-ya Ko ${ }^{3}$, and \\ Chen-te $\mathrm{Yao}^{3}$ \\ ${ }^{1}$ National Taiwan Normal University \\ ${ }^{2}$ Sun Yat-sen University \\ ${ }^{3}$ Affiliation not available \\ ${ }^{4}$ Endemic Species Research Institute
}

July 12, 2021

\begin{abstract}
The long-term persistence of a population which has suffered a bottleneck partly depends on how historical demographic dynamics impacted its genetic diversity and the accumulation of deleterious mutations. Here we provide genomic evidence for the detrimental genetic effect of a recent population bottleneck in the endangered black-faced spoonbill (Platalea minor) even after its rapid population recovery. Our population genomic data suggest that the bird's effective population size, $N_{e}$, had been relatively stable $(7,500-9,000)$ since the end of the last glacial maximum; however, a recent brief yet severe bottleneck $\left(N_{e}=20\right)$ around the 1940 s wiped out more than $99 \%$ of its historical $N_{e}$ in roughly three generations. By comparing it with its sister species, the royal spoonbill ( $P$. regia) whose conservation status is of lesser concern, we found that despite a more than 15 -fold population recovery since 1988, genetic drift has led to higher levels of inbreeding (7.4 times more runs of homozygosity longer than $100 \mathrm{~Kb}$ ) in the black-faced spoonbill than in the royal spoonbill genome. Although the two spoonbills have similar levels of genome-wide nucleotide diversity and heterozygosity, because of relaxed purifying selection, individual black-faced spoonbills carry $3 \%$ more nonsynonymous substitutions than royal spoonbills each of which is $7 \%$ more deleterious. Our results imply that the persistence of a threatened species cannot be inferred from a recovery in its population. They also highlight the necessity of continually using genomic indices to monitor its genetic health and employing all possible measures to assure its long-term persistence in the ever-changing environment.
\end{abstract}

\section{Introduction}

Human activities are driving up to one million species to extinction(IPBES, 2019; Pimm et al., 2014). All threatened species are characterized by small population sizes and a declining population trend or have been through population bottlenecks. Theories suggest that when a population becomes small or has gone through a population bottleneck, the drastically augmented random fluctuation of allele frequencies over time (genetic drift) could lead to the loss of its genetic variation, i.e. genetic erosion. This would compromise a species' potential to evolve in response to the ever-changing environment(Barrett \& Schluter, 2008; Lai et al., 2019; Lande \& Shannon, 1996; Bijlsma \& Loeschcke, 2012), and lower the efficacy of purifying selection in removing deleterious genetic variants(Kimura, 1962; Kirkpatrick \& Jarne, 2000) resulting in the accumulation of deleterious mutations in it (mutation load(Kimura, 1962; Kimura, Maruyama, \& Crow, 1963). It can also elevate levels of inbreeding that increase homozygosity and the expression of deleterious recessive alleles, thereby reducing individual fitness (Charlesworth \& Charlesworth, 1987; Keller \& Waller, 
2002). Moreover, theories suggest that such detrimental consequences may persist even after a population re-expands (Kirkpatrick \& Jarne, 2000). Therefore, further conservation measures would be required to assure the persistence of a re-expanded endangered population. Although a low level of genetic variation and accumulated deleterious mutations were found in endangered or bottlenecked populations (Robinson et al., 2016; Grossen, Guillaume, Keller, \& Croll, 2020), results of some genomic studies suggest otherwise: bottlenecked non-African human populations do not have lower genetic variation, and the effectiveness of purifying selection to remove deleterious mutations is not compromised in the European population (Do et al., 2015; Fu, Gittelman, Bamshad, \& Akey, 2014; Lohmueller, 2014; Simons, Turchin, Pritchard, \& Sella, 2014). This implies that further conservation provisions might not be essential for the long-term survival of a bottlenecked population. Therefore, the ability of a threatened species to persist may partly depend on the extent to which the historical bottleneck event caused a decay in its genetic diversity and an increase in its mutation load.

The once critically endangered migratory wader, the black-faced spoonbill (Platalea minor )(BirdLife International, 2017), could serve as an ideal system to assess the genetic legacy of a bottleneck event in a re-expanding population. $P$ minor winters in the coastal salty wetland habitats of the East Asian coast (Fig. 1A). Its extant breeding colonies are mainly located on uninhabited rocky islets along the western and eastern coast of the Korean Bay (Chong, Pak, Rim, \& Kim, 1996; Ding, Lei, Yin, \& Liu, 1999; Ueta et al., 2002), and recently expanded to the coast along the Northern Sea of the Japan Basin (Litvinenko \& Shibaev, 2005; Shibaev, 2010). This species was described as 'common' in documents pre-dating the 1950s (La Touche, 1931; Austin, 1948). However, only 288 individuals were counted within its entire range in 1988 (Kennerley, 1990). Since then, its population size has increased remarkably to 4,864 individuals according to a census in the winter of 2020 (Yu, Li, Tse, \& Fong, 2020). A study of mitochondrial diversity suggested that the spoonbill had recently experienced a severe bottleneck (Yeung, Yao, Hsu, Wang, \& Li, 2006). However, without a documented history of the timing, magnitude and duration of the bottleneck, we cannot rule out the possibility that its low population size in the 1980s was part of a natural response to the drastic climate changes since the end of the last glacial maximum (LGM), as in some other endangered species (Mays et al., 2018).

To infer the timing and duration of its presumed recent bottleneck event, we first sequenced and obtained a draft assembly of the black-faced spoonbill genome, then re-sequenced the whole-genome of multiple individuals collected from its two major wintering sites in Taiwan and Hong Kong (Yu et al., 2020) (Fig. 1A). We evaluated the extent of genetic diversity and deleterious mutations accumulated in the extant blackfaced spoonbill population by comparing its population genomic data to that of its sister species the royal spoonbill ( . regia ) from which it diverged approximately half a million years ago ( Yeung et al., 2011). The royal spoonbill is commonly found in the wetlands of Australia and nearby islands (Fig. 1A), and its conservation status is in the 'least concern' category; it does not have a documented history of bottlenecks (Matheu \& del Hoyo, 1992). We specifically addressed the following for the black-faced spoonbill: (1) whether the drastic climate change since the end of the LGM significantly impacted its population trajectory before the presumed recent population bottleneck; (2) what the start time, duration and magnitude of the presumed recent bottleneck were; (3) whether the recent bottleneck event, if any, has led to a relaxation of selection and a higher level of inbreeding, genetic drift and the accumulation of deleterious mutations than in the royal spoonbill.

\section{Results}

\section{De Novo assembly of a draft genome for the black-faced spoonbill}

We sequenced and conducted a de novo genome assembly to generate a reference genome from a rescued male black-faced spoonbill. The assembled genome is $1.19 \mathrm{~Gb}$ in length (genomic coverage $=42.5 \times$ ) and contains 2,243 scaffolds ( 870 scaffolds > $10 \mathrm{~Kb}$, total $1.186 \mathrm{~Gb}$; scaffold N50 of $4.2 \mathrm{Mb}$; 34,176 contigs; contigs N50 of $71.0 \mathrm{~Kb}$; Table S1). This draft genome includes a total of $78.2 \mathrm{Mb}(6.6 \%$ of total genome) of repetitive sequences (Table S2). We found 25,315 predicted genes, 11,733 of which could be functionally annotated. In total, we obtained $399.6 \mathrm{Mb}$ of genic regions (33.6\% of the total genome) consisting of $365.2 \mathrm{Mb}$ of introns 
and 34.4 Mb of coding regions. The completeness of our draft genome is high (Table S3): we identified 93.0\% $(4,569)$ of the Benchmarking Universal Single-Copy Orthologs v3.0(Waterhouse et al., 2018). Therefore, the completeness of our draft genome is comparable to other published avian genomes, such as the draft genome of horn lark (Mason, Pulgarin, Cadena, \& Lovette, 2020).

\section{Comparable genomic diversity between the black-faced spoonbill and royal spoonbill}

We re-sequenced the genomes of 11 black-faced spoonbill individuals, nine of which were from Tainan County, Taiwan, the wintering ground of more than $50 \%$ of the species' world population (Chesser, Yeung, Yao, Tian, $\&$ Li, 2010) and two from the Deep Bay Wetland, Hong Kong (Fig. 1A; Table S4). We also used the draft genome of the black-faced spoonbill as the reference to re-sequence the whole genomes of nine wild royal spoonbill individuals from Australia (Table S4). The genomic diversities of the two spoonbill species are comparable: the nucleotide diversity $(\pi)$ and mean individual heterozygosity of the black-faced spoonbill are $1.11 \times 10^{-3}$ and $1.09 \times 10^{-3}$, respectively. The comparable figures are $1.06 \times 10^{-3}$ and $1.05 \times 10^{-3}$ in the royal spoonbill (Table S5). The genome-wide heterozygosities of both spoonbill species are relatively low (Table S6) compared with those of most of the 32 avian species (Lai et al., 2019; Li et al., 2014) in the "Least Concern" category in the IUCN Red List (mean \pm S.D. $\left.=2.50 \pm 1.07 \times 10^{-3}\right)$, but twice that of the critically endangered crested ibis (Nipponia nippon (S. Li et al., 2014); $=0.43 \times 10^{-3}$ ), and close to that of seven vulnerable species(S. Li et al., 2014) $\left(1.18 \pm 0.85 \times 10^{-3}\right)$. Our results suggest that the genetic diversity of black-faced spoonbills is still comparable to those of the un-threatened royal spoonbill and other vulnerable but not endangered avian species. The genome-wide genetic distance $\left(D_{\mathrm{xy}}\right)$ between the two spoonbills was estimated to be 0.0020 . This is consistent with the short mitochondrial DNA distance (the Tamura-Nei distance $=0.015$ ) observed between these two sister species (Chesser et al., 2010).

\section{Genetic panmixia of black-faced spoonbills sampled in Taiwan and Hong Kong}

Migratory birds with different wintering grounds might have fidelity to their own breeding grounds which could lead to genetic differentiation (Rubenstein et al., 2002). To test whether black-faced spoonbills wintering in Taiwan and Hong Kong originated from different genetic groups, we conducted a model-based admixture analysis (Alexander \& Lange, 2011) based on 215,722 unlinked $\left(r^{2}<0.2\right)$ genome-wide autosomal SNPs (coverage $>10 \times$ ). The results of the admixture analysis (Fig. 1B) and principal components analysis (Fig. 1C) suggest that all black-faced spoonbill individuals sampled in the current study likely consisted of a single breeding population, allowing us to combine the genomic data from the individuals from the two wintering sites for the subsequent analyses. Our results are consistent with satellite tracking records which showed black-faced spoonbills tagged in both Taiwan and Hong Kong returning to the Demilitarized Zone of Korea in the breeding season (Ueta et al., 2002).

\section{Relatively stable post-LGM demographic histories of the black-faced and royal spoonbills}

We reconstructed the historical demographic trajectories of the two sister spoonbill species with $S M C++$ (Terhorst, Kamm, \& Song, 2017). The $N$ e of the black-faced spoonbill was estimated to be about 8,900 individuals at the LGM (approximately 22,000 years ago), which then gradually declined to 7,432 individuals until around 435 years ago (Fig. 2A). Conversely, the royal spoonbill had $a n N$ e that gradually increased from approximately 7,800 individuals at the LGM to 8,130 individuals around 500 years ago (Fig. 2A). The similar post-LGM $N$ e trajectories of the two species also make the royal spoonbill an ideal control group to infer the effect of a recent bottleneck on the genome of the black-faced spoonbill.

\section{A brief yet severe recent bottleneck for the black-faced spoonbill}

To test whether the black-faced spoonbill experienced a recent bottleneck event, we compared the maximum composite likelihood of a recent bottleneck model and a constant population size model based on 215,722 unlinked autosomal SNPs using Fastsimcoal2 (Excoffier, Dupanloup, Huerta-Sánchez, Sousa, \& Foll, 2013). The results significantly supported the recent bottleneck model (maximum estimated likelihood $=-230,635.54$, $\mathrm{AIC}=421,681.07$, AIC weight $=1.0$ ) instead of a constant population model (maximum estimated likelihood= -232,073.84; $\mathrm{AIC}=464,149.07, \Delta \mathrm{AIC}=2,868.6, \mathrm{AIC}$ weight $=0.0)$. The presumed bottleneck event was 
estimated to be very recent and brief (Fig. 2B), starting approximately six generations ago (95\% CI: 4-12 generations ago) and ending about three generations ago (95\% credible interval, CI: 2-4 generations ago). Because most black-faced spoonbill individuals (9 of 12 samples) sequenced in this study were victims of a botulism event in 2002, and assuming a generation time of 10 years (Yeung et al., 2006), our results suggest that the recent bottleneck event occurred around the 1940s.

\section{An elevated level of inbreeding in the black-faced spoonbill}

Small populations are particularly prone to an elevated level of inbreeding, the mating between relatives, which can increase homozygosity at loci that carry deleterious recessive mutations and thus reduce the fitness of offspring (Crow \& Kimura, 1970) (i.e. inbreeding depression). Two lines of evidence reveal the genomic footprints of inbreeding during the bottleneck and possibly the post-bottleneck period in the blackfaced spoonbill population. A recent and severe bottleneck event is expected to result in an increase in the number $(\mathrm{NROH})$ and length $(\mathrm{SROH})$ of runs of homozygosity, which are identical homologous genomic segments(McQuillan et al., 2008). We found that the black-faced spoonbill had approximately 7.4 times the SROH $(t$ test $=-12.68, p<0.0001)$ and 6.2 times the NROH $(t$ test $=-22.78, p<0.0001)$ of the royal spoonbill (Fig. 3A; Table S7): the black-faced spoonbill had an average NROH of $426.42 \pm 49.65$ and average $\mathrm{SROH}$ of $84,154.15 \pm 19,442.36 \mathrm{~Kb}$; by contrast, the royal spoonbill had an average $\mathrm{NROH}$ of $68.89 \pm 19.21$ and an average $\mathrm{SROH}$ of $11361.82 \pm 3645.35 \mathrm{~Kb}$. In both species, most of the ROHs were less than $500 \mathrm{~Kb}$ long $(84.6 \%$ and $90.4 \%$ of SROH for the black-faced and royal spoonbill, respectively; $96.3 \%$ and $97.6 \%$ of $\mathrm{NROH}$ for the black-faced and royal spoonbills, respectively), yet significantly longer ROHs $(>2,000 \mathrm{~Kb})$ were observed in two black-faced spoonbills with the longest being 3,566.6 Kb. The genomic inbreeding coefficient, $F_{\text {ROH }}$, which measures the fraction of the autosomal genome in ROH ( Keller, Visscher, \& Goddard, 2011), was approximately seven times higher in the black-faced spoonbill than in the royal spoonbill (mean $F$ $\mathrm{ROH}=0.071$ and 0.001 for the black-faced and royal spoonbills, respectively), but lower than those of other threatened populations (e.g. $F_{\mathrm{ROH}}=0.4$ in the grey wolf (Gómez-Sanchez et al., 2018)). This may reflect alleviated inbreeding due to the rapid population recovery. Moreover, inbreeding could also lead to a higher level of homozygosity and slower decay in linkage disequilibrium (Slatkin, 2008) which can be measured by the association of occurrence of alleles at two loci, expressed as a correlation coefficient $\left(r^{2}\right)$. In this case, $r$ ${ }^{2}$ decay was slower in the black-faced spoonbill than in the royal spoonbill (Fig. 3B).

\section{An elevated level of genetic drift in the black-faced spoonbill}

A bottleneck event could intensify the effect of genetic drift, resulting in an increased rate of extinction and therefore a deficiency of low-frequency alleles as well as an over-abundance of medium frequency alleles (Tajima, 1989; Gattepaille, Jakobsson, \& Blum, 2013; Maruyama \& Fuerst, 1985). To measure the strength of genetic drift in the black-faced spoonbill, we calculated the folded site frequency spectrum (SFS) of unlinked SNPs of the autosomal chromosomes for the two spoonbill species. The folded SFS of the two spoonbill species were bimodal distributions (Fig. 3C) with a tall peak for singletons (minor SNPs carried by only one haplotype) and a lower peak for medium-frequency SNPs. However, the frequency of singletons in the black-faced spoonbill (21.9\%; 47,227 of 215,722 SNPs) was significantly lower than that in the royal spoonbill $\left(30.7 \% ; \chi^{2}=24.82, p=0.000\right)$. In theory, genome-wide Tajima's $D$ values would be higher with fewer low-frequency SNPs (Tajima, 1989) (e.g. singletons), as was the case in the black-faced spoonbill (mean Tajima's $D=0.24 \pm 0.98$, the number of total SNPs, $n=4,522,032$ ) compared with the royal spoonbill (mean Tajima's $D=0.05 \pm 0.99, n=4,437,044 ; t=296.51, p<0.001 ;$ Fig. 3D). The lower singleton frequency and higher Tajima's $D$ values suggest that the black-faced spoonbill has been subjected to stronger genetic drift caused by the recent population bottleneck.

\section{Relaxation of selection in the black-faced spoonbill}

The elevated level of genetic drift in the black-faced spoonbill might have compromised the efficacy of natural selection (Kimura, 1962). To examine whether the elevated level of genetic drift had lowered selection efficiency in the black-faced spoonbill, we used RELAX (Wertheim, Murrell, Smith, Kosakovsky Pond, \& Scheffler, 2015) to detect genes under relaxed selection in the two spoonbills. Of the 5,657 well-aligned 
homologous genes between the two spoonbill species and another Ciconiformes species, the crested ibis ( Li et al., 2014), RELAX analysis revealed significantly more genes in the black-faced spoonbill $(2,094,37.0 \%)$ to be under relaxed selection (relaxation parameter $k<1, p<0.05)$ than in the royal spoonbill $(1,542,27.2 \%$; $\chi^{2}=123.04, p=2.2 \mathrm{e}^{-16}$; Figure $\left.4 \mathrm{~A}\right)$. Conversely, there was no evidence $\left(\chi^{2}=3.1671 .04, p=0.075\right)$ to support the hypothesis that selection was significantly intensified in the genome of the royal spoonbill (33 and 55 genes with $k>1, p<0.05$ for the royal and black-faced spoonbill, respectively; Figure 4A). Therefore, a higher level of genetic drift had probably caused relaxed selection in the black-faced spoonbill.

\section{Higher mutation load in the black-faced spoonbill}

A consequence of relaxed selection is the accumulation of deleterious mutations in the genome (Kimura, 1962; Kirkpatrick \& Jarne, 2000). We found that black-faced spoonbill individuals carried about 3\% (8,294.00土 332.66 substitutions) more nonsynonymous substitutions (NS) than royal spoonbill individuals $(8,025.78 \pm$ 211.77 substitutions; $t=-1.91, p<0.035$; Fig. 4B; Table S8). Moreover, the mean phenotypic effect, Grantham's score (Grantham, 1974), for each deleterious NS (Grantham's score> 50; Chun \& Fay, 2009)) was approximately $7 \%$ higher for the black-faced spoonbill (56.98 \pm 0.69$)$ than in the royal spoonbill ( $53.25 \pm 1.83$; two-sample $t$ test $=6.5154, p=3.6 \mathrm{e}^{-6}$; Table S9). As a result, the average accumulated Grantham's score per individual was higher in the black-faced spoonbill $(527,350 \pm 33,698.9)$ than in the royal spoonbill $(502,030 \pm$ $9,571.9 ; t=2.47, p=0.014$; Fig. $4 \mathrm{C}$; Table S9). Moreover, the ratio of homozygous to heterozygous NS in the black-faced spoonbill $(0.13 \pm 0.04)$ was also significantly higher than that in the royal spoonbill $(0.11 \pm$ $0.007 ; t=-1.86, p<0.04$; Fig. 4D), suggesting that purifying selection has been less effective in removing deleterious homozygotes in the bottlenecked black-faced spoonbill. Therefore, we provide a real-world example of the theory that mutation load, accumulation of deleterious mutations, is insensitive to recent population growth (Simons et al., 2014).

\section{Discussion}

Relatively stable post-LGM demography

The historical demographic trajectory retrieved from population genomic data (Fig. 2A) suggests that the $N$ e of the this once critically endangered species is much larger than any census global population sizes since 1988, and was stable for most of its recent evolutionary histories, unperturbed by the drastic climatic changes since the end of the LGM. The large and stable historical $N_{\mathrm{e}}$ of the black-faced faced spoonbill is consistent with the fact that its genome-wide genetic diversity ( $\pi$ and mean individual heterozygosity) is similar to that of the royal spoonbill which also had a similar post-LGM demographic trajectory (Fig. 2A).

Another line of evidence which supports the historical abundance of the black-faced spoonbill as documented in early literature (La Touche, 1931; Austin, 1948) is the fact that that its estimated long-term $N_{e}$ could be translated, assuming a simple one-to-ten rule for the ratio between $N_{e}$ and the census breeding population size (Frankham, 1995), into over 74,200 breeding individuals. This is comparable to the similarly deduced long-term breeding population size of the royal spoonbill of 78,000 individuals and to the current population size of royal spoonbills (25,000-100,000 individuals; Matheu \& del Hoyo, 1992). A stable and large long-term $N_{e}$ rules out the possibility that the bottleneck observed in 1988 was the result of the long term population decline driven by climate change since the LGM as in the case of other endangered species (Mays et al., 2018; Li et al., 2014; Cho et al., 2013; Zhao et al., 2013).

Could DDT have been the cause of recent population bottleneck?

Although we cannot ascertain the cause of this recent bottleneck, its estimated start coincides with the period of the widespread use of organochlorine pesticides (e.g. DDT) around the world, including East Asia (Wong, Leung, Chan, \& Choi, 2005), after World War II. DDT and its metabolites are known to impair the reproduction of avian species by thinning eggshells and acting as estrogen blockers (Cooke, 1973; Risebrough, 1986). DDT-induced population crashes have been documented in several raptors (Poole, 1989; Newton \& Wyllie, 1992); eggshells of other Ciconiformes species (Faber \& Hickey, 1973; King, Meeker, \& Swineford, 1980) were found to thin significantly in the presence of even a miniscule amount of DDT. Furthermore, the 
black-faced spoonbill is one of the top predator species in its major wintering habitat, the estuaries, which are known to be repositories of contaminants (Ridgway \& Shimmield, 2002) where biomagnification through the food chain would expose the bird to a level of DDT and its metabolites higher than that found in the environment. The adverse effects of DDT on wildlife eventually led to its ban around the world (e.g. in 1972 by the United States), and has been associated with the rapid recovery of some raptors (Grier, 1982). The bottleneck event of the black-faced spoonbill was estimated to have ended two to three generations ago, which is around 1970-1980s, consistent with the prohibition of the use of DDT in East Asia in the 1980s (China in 1983, South Korea in 1986, and Taiwan in 1987) and the black-faced spoonbill's population growth trend since 1988 .

The recent bottleneck event experienced by the black-faced spoonbill was, although brief, extremely severe:

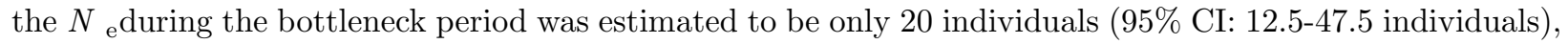
roughly equivalent to 200 breeding individuals. By contrast, the pre-bottleneck $N$ ewas estimated to be 5491.5 (95\% CI: $4549.5-12310.5$ individuals), close to the recent historical $N_{\text {e }}(7,432)$ estimated by $S M C++$. In other words, more than $99 \%$ of $N$ e vanished during the bottleneck event. Although the $N_{\mathrm{e}}$ of the black-faced spoonbill has since increased to 236.5 (95\% CI: 57.6 - 376.0), it is still only about $4 \%$ of its pre-bottleneck $N$ e. The magnitude of $N$ e reduction would rule out the Korean War as a candidate cause of population decline (Matheu \& del Hoyo, 1992) since the uninhabited rocky islets where the bird breeds would hardly have been major military targets.

The questionable long-term persistence of the black-faced spoonbill

The rapid population recovery of the black-faced spoonbill may preclude the risk of immediate extinction, although whether its current $N$ e of 405 could support the species' long-term persistence remains questionable, as it has been suggested that a minimum $N$ e of 1,000 is required to ensure the evolutionary potential and long-term persistence of a population (Frankham, Bradshaw, \& Brook, 2014). Moreover, the coastal wetlands of East Asia are under unprecedented pressure of reclamation, urbanization and industrialization (Larson, 2015). For example, a seawall in China which encloses its coastal wetland to support rapid urbanization and economic development reached 11,000 km (70\% of mainland China's coastline) in 2010 (Ma et al., 2014), fundamentally altering the coastal ecosystem and inevitably reducing the environmental carrying capacity for the black-faced spoonbill to a level much lower than that in the pre-bottleneck era. The deterioration of its wintering habitat is unlikely to have been responsible for the past bottleneck event because the habitat has deteriorated continually, which is inconsistent with the recent population recovery (Baillie, Hilton-Taylor, \& Stuart, 2004). Habitat deterioration would reduce the environmental carrying capacity of the black-faced spoonbill and constrain the recovery potential and persistence of this endangered species. Unless stringent conservation measures are taken to improve or at least maintain its current carrying capacity, the future of the black-faced spoonbill is still in doubt despite its recent rapid population growth (Sung, Tse, \& Yu, 2018).

Although our results support the conclusion that the black-faced spoonbill experienced a recent severe population bottleneck, its genome-wide genetic diversity ( $\pi$ and mean individual heterozygosity) is comparable to that of the royal spoonbill and other non-vulnerable avian species (Table S5, S6). This is probably because the short bottleneck duration and the rapid population recovery only led to the loss of rare genetic variants. This is what is suggested by the lower frequency of singleton SNPs in the folded site frequency spectrum (Fig. 3C) and higher genome-wide Tajima's $D(>0)$ values in the black-faced spoonbill. This observation is consistent with a simulation study: a short bottleneck is less likely to cause severe genetic erosion (Allendorf, 1986). Since standing genetic variation with variants present at high frequencies could allow a species to respond more swiftly to selection pressures posed by changing environments (Hermisson \& Pennings, 2005; Lande \& Shannon, 1996), our results suggest that the recent drastic population bottleneck event might not severely dampen the evolutionary potential of the black-faced spoonbill. Therefore, if its further population recovery can be sustained, the chance for this once critically endangered wader to cope with future environmental changes should be approximately the same as for royal spoonbills and other non-vulnerable species.

Although the extant black-faced spoonbill might not be suffering from severe genetic erosion, it could still 
be the victim of an elevated level of inbreeding (Fig. 3) and mutation load (Fig. 4). However, despite the fact that these birds carry higher numbers of detrimental mutations, its population has still grown steadily since 1988 (Yu et al., 2020). One possible explanation for this is that the fitness effect of mutations could be environment-dependent (Szafraniec, Borts, \& Korona, 2001): the high genetic load in the blackfaced spoonbill might not necessarily confer lower population fitness in a benign environment. However, when the environment becomes more stressful, the impact of mutations' deleterious effects on fitness could increase (Martin \& Lenormand, 2006), especially when exacerbated anthropogenic environmental change is expected to further reduce the habitat quality of wildlife for the foreseeable future. Therefore, even the blackfaced spoonbill population rebound led to its being down-listed from critically endangered to endangered in the IUCN Red List, and is considered a success example of international collaborative conservation efforts (BirdLife International, 2000), our study suggests that these birds are not totally out of the woods yet.

Furthermore, our results provide an example of modern genomic sequencing technologies as a powerful tool to monitor the genetic health of and predict the evolutionary potential for any species whose conservation is of concern. In the case of black-faced spoonbills, genomic data suggest that even after decades of rapid population rebound, its fate is still in the mists and further conversation measures are needed to assure its survival given that its habitat will inevitably be subject to tremendous anthropogenic impacts. Given both theoretical (Robertson \& Waddington, 1960; Hill \& Robertson, 1966) and empirical (Weber, 2004) works suggesting that selection is more effective in larger populations, rapid population recovery after a bottleneck event may be critical to restore the efficacy of selection to purge out accumulated detrimental mutations. Aiding the re-expansion of the black-faced spoonbill should be considered as an overarching objective of its conservation management in near future. All conservation practices, such as habitat restoration (Dobson, Bradshaw, \& Baker, 1997), supplementary feeding (Ewen, Walker, Canessa, \& Groombridge, 2015), predator removal (Smith, Pullin, Stewart, \& Sutherland, 2010), and population augmentation from ex situ population(Bowkett, 2009) should be seriously considered and employed. In addition, we propose that the level of inbreeding and mutation load of black-faced spoonbill individuals should be continually monitored. Such information would provide temporal genomic indices (such as $\mathrm{ROH}$ ) to evaluate how its genetic health has been improved by current conservation practices to assist its population recovery strategy. Taken together, our results are especially relevant when anthropogenic environmental changes are increasingly turning incidental natural processes into catastrophic events which put many wildlife species in great peril of population reduction.

\section{Acknowledgements}

We thank Terry Chesser and the Commonwealth Scientific and Industrial Research Organization of Australia for providing samples of the royal spoonbills and Carrie K.W. Ma for providing samplings of black-faced spoonbills from Hong Kong. We are grateful for the valuable help and comments by Chih-Ming Hong, Wei Liang, Teng-Chiu Lin, and Benyang Liao. We are in debt to Alan Watson who has greatly improved the readability of this manuscript. This work was supported by grants to C.- T. Yao from the Taiwan Endemic Species Research Institute and to S.-H. Li from the Ministry of Science and Technology, Taiwan, R.O.C., and Y. Liu by Forestry Administration of Guangdong Province, China (DFGP Project of Fauna of Guangdong-202115. It was also kindly supported by endorsement from Mr. Y.-C. Chen.

Author contributions : S.-H.L., C.-T.Y. and Y.L. designed research; C.-F.Y., and Y.C.C. performed research; W.-Y.K., T.H.K, and F.-T. C. contributed new reagents/analytic tools; C.-F.Y., C.C.C., , and Y.C.C., analyzed data; and S.-H.L., Y.L. C.K.L.Y., C.-C.L., Y.F., and C.-T.Y. wrote the paper. All authors approved the final submission.

Competing interests: The authors declare that they have no competing interests.

\section{Materials and Methods}

\section{Reference genome}

Sampling, DNA extraction, Genomic library preparation and sequencing 
In 2015 we used a modified chloroform and LiCl precipitation protocol(Gemmell \& Akiyama, 1996) to extract gross DNA from the blood of a rescued male black-faced spoonbill $\left(22.88^{\circ} \mathrm{N}\right.$ and $\left.120.20^{\circ} \mathrm{E}\right)$ which was kept in the Taiwan Endangered Species Research Institute.

Libraries with insert size $200 \mathrm{bp}, 3 \mathrm{~Kb}$ and $5 \mathrm{~Kb}$ were constructed (with the Paired-End DNA Sample Prep Kit for the $200 \mathrm{bp}$ library and Mate Pair Library Prep Kit v2 for the $3 \mathrm{~Kb}$ and $5 \mathrm{~Kb}$ libraries) and sequenced on an Illumina HiSeq 2500 platform by Genomics BioSci \& Tech (Taipei, Taiwan). In total, 124 Gb raw reads were generated (table $\mathrm{S} 1$ ).

\section{Assembling the reference genome de novo}

We used ALLPATHS-LG version-44099(Gnerre et al., 2011) (parameters PLOIDY=2 and PHRED_64=1) to assemble the draft genome of the black-faced spoonbill. We ran ALLPATHS-LG on a workstation with 32 CPUs $(2,199.882 \mathrm{MHz}$ ) and 377.8 Gb RAM. (The peak value of in-use memory was $342.1 \mathrm{~Gb}$.)

We used the correction steps CleanCorrectedReads andErrorCorrectJump in ALLPATHS-LG to remove $1.7 \%$ of paired-end reads and to correct $69.3 \%$ of mate-paired reads with the criterion of low frequency k-mers ( $\mathrm{K}=25$ and 96 for paired-end and mate-paired reads respectively). The raw reads for assembling were $50.5 \mathrm{~Gb}(67.8 \%)$ and $28.8 \mathrm{~Gb}(57.5 \%)$ of paired-end and mate-paired reads, respectively (table S1). In total 34,176 contigs with N50 size of $71.0 \mathrm{~kb}$ were assembled with a total length of $1.18 \mathrm{~Gb}$. Finally, 2,243 scaffolds $(\mathrm{N} 50=4.2 \mathrm{Mb})$ (table $\mathrm{S} 1)$ were concatenated from these contigs. The draft genome generated by $A L L P A T H S-L G$ is a diploid genome. For subsequent analyses, we randomly dropped one of the nucleotides from each heterozygous SNP to generate a pseudo-haploid reference genome.

Masking repetitive sequences

We masked repetitive sequences from the draft genome withRepeatMasker version open-4.0.7, with the options -gccalc and -species "aves." In total we masked 78.2 Mb (6.6\%) of the genome sequence.

\section{Removing contaminating sequences}

We removed potential contaminating sequences in the draft genome by following the procedure in Jones et al.(Jones et al., 2012). On each scaffold, a $1 \mathrm{~Kb}$ fragment without an unknown base "N" was sampled from each $100 \mathrm{~Kb}$ window to check for the presence of contaminants. In total we sampled 13,105 one kb fragments and blasted them against the NCBInt database with $B L A S T N$ (v. 2.6.0; e-value cut-off $=10^{-5}$ ). Fragments of the $B L A S T N$ ' s top hit species that were neither birds nor reptiles were considered contaminants. We extracted contigs that contained these fragments from the scaffolds. In total we found contaminated DNA in 9 scaffolds $(153.8 \mathrm{Mb})$, which included bacteria $(\mathrm{N}=2)$, fishes $(\mathrm{N}=1)$ and mammals $(\mathrm{N}=6)$. Finally, 2,243 scaffolds were retained in the draft black-faced spoonbill genome assembly.

\section{Constructing the putative chromosome}

To infer the putative autosome of the black-faced spoonbill, we usedSatsuma v2.1(Grabherr et al., 2010) to align our reference genome assembly to the chicken genome (GRCg6a; GCA_000002315.5(Warren et al., $2017)$ ) with option "l (minimum alignment length) $=100$ ". Scaffolds embedded within other scaffolds were removed. If the same scaffold was repeatedly aligned to different chromosomes, we kept the longest alignment. 486 scaffolds with a total length $1.10 \mathrm{~Gb}$ (93\% of total draft genome) of the black-faced spoonbill genome were aligned to seven macro-chromosomes, 30 intermediate and micro-chromosomes and the mitochondrial DNA of the chicken genome. We classified unaligned scaffolds as "unlocalized" (Table S4).

\section{Genome annotation}

\section{Gene structure and functional annotation}

To annotate the draft genome of the black-faced spoonbill, we used RNeasy Mini Kit to extract total RNA from a blood sample from the same individual used for reference genome sequencing following the manufacturer's instructions. RNA was sequenced by Genomics BioSci \& Tech (Taipei, Taiwan) using a TruSeq Stranded mRNA Preparation Kit on an Illumina NextSeq 500 platform with the average insert size 
of $180 \mathrm{bp}$. We used Trinity v 2.4.0(Grabherr et al., 2011) to analyze 5.2 GB of RNA-seq reads. We used both de novo and genome guide (using the draft genome assembly as a reference) methods to generate $273 \mathrm{Mb}$ and $171 \mathrm{Mb}$ of RNA assembly, respectively. Then, the two assemblies were merged with PASA (Haas et al., 2003). TransDecoder in PASA was used to identify likely coding regions (CDS) with the CDS of the genome of the crested ibis Nippon nippon(GCF_000708225.1_ASM70822v1(S. Li et al., 2014)) as the reference. Finally, we obtained $190 \mathrm{Mb}$ of spoonbill RNA assembly as the gene model to train Augustus v3.2.3 (Stanke \& Morgenstern, 2005) for gene prediction. Augustus predicted 11,733 genes totaling $411 \mathrm{Mb}$ (35\% of the total genome) and $25 \mathrm{Mb}$ of CDS in the spoonbill genome. We extracted the coding regions of all annotated genes and blasted these CDS to Gallus_gallus-5.0 (GCF_000002315.4) with BLASTP (v. 2.6.0; e-value cut-off= $\left.10^{-6}\right)$. There were only 10,558 genes that met the e-value cut-off criterion.

Evaluating the completeness of the draft genome

We used 4,915 Benchmarking Universal Single-Copy Orthologs (BUSCO v3.0.1, (Waterhouse et al., 2018)) to evaluate the completeness of the genome assembly and annotation.

\section{Population re-sequencing}

Population sampling and whole genome resequencing

In addition to the sample used for reference genome sequencing, we also re-sequenced eleven black-faced spoonbills whose tissue was collected from individuals that had been rescued or had accidentally died in Tainan County, Taiwan $(\mathrm{N}=)$ and the Deep Bay Wetland, Hong Kong $(\mathrm{N}=2)$, as well as tissue samples of royal spoonbills from Australia $(\mathrm{N}=9)$.

We used a protocol from Gemmel and Akiyam (2006) to extract gross DNA from these tissue samples for resequencing. A whole genome re-sequencing library with insert size 50 bp was constructed for each individual. Our sequencing data was generated using an Illumina HiSeq 4000 platform (BGI).

Re-sequencing mapping and variant calling

A consensus sequence for each individual was generated using Vcf-tools v.0.1.13(Danecek et al., 2011). The average coverage of the black-faced and royal spoonbills was $15.7 \times$ and $16.6 \times$, respectively.

\section{Population structure}

Two approaches were used to examine the population structure of the black-faced spoonbill as sampled from Taiwan and Hong Kong. Firstly, we used ADMIXTURE v1.22(Alexander \& Lange, 2011), which is based on a Bayesian clustering method, to infer the number of ancestors of the black-faced spoonbills sampled in current study. Only unlinked autosomal SNPs were used for the admixture analysis. We used PLINK (Purcell et al., 2007) to filter out linked autosomal SNPs. The window size to calculate pair-wise SNP-SNP $r^{2}$ was set to $10 \mathrm{~Kb}$, and the step of the sliding window was set to $2 \mathrm{~Kb}$. The threshold of $r^{2}$ was set to 0.2. SNPs that significantly deviated from the Hardy-Weinberg expectation $(p<0.001)$ were also filtered out. In total 25,172 unlinked autosomal SNPs $\left(r^{2}<0.2\right)$ with $>10 \times$ coverage were used in the admixture analysis.

Secondly, we used GCTA (Yang, Lee, Goddard, \& Visscher, 2011) to carry out principal components analysis (PCA). The same set of 25,172 unlinked autosomal SNPs was analyzed. The score of the first component (PC1; $1.33 \%$ of variance) was plotted against the score of the second component ( $\mathrm{PC} 2 ; 1.28 \%$ of variance) of each individual to illustrate the genetic affinity between the black-faced spoonbill samples.

\section{Historical demography}

\section{Long-term historical demography}

To infer the historical demography of the black-faced spoonbill, we applied $S M C++$ (Terhorst et al., 2017) which employs a coalescent-based method utilizing information on the site frequency spectrum and linkage disequilibrium of a set of diploid genomes to infer the historical demographic dynamics of the population concerned. To enhance base calling accuracy, we used the VCF containing diploid genotypes generated from 
all individuals $(\mathrm{N}=9)$ with coverage greater than 15 for the $S M C++$ analysis. We used the command $-\mathrm{CV}$ to perform cross-validation which yielded model parameters for calculating the effective population size through time. The generation time of the black-faced spoonbill was set to ten, and the mutation rate was set to $3.6 \times$ $10^{-8}$ per site per generation(Axelsson, Smith, Sundström, Berlin, \& Ellegren, 2004). We also used SMC ++ to infer the historical demography of the royal spoonbill based on diploid genotypes with coverage greater than $15(\mathrm{~N}=7)$.

\section{Detection of a recent bottleneck}

We used a model-selection approach to detect whether the black-faced spoonbill had experienced a recent population bottleneck. Two demographic models were constructed for the recent demography of the blackfaced spoonbill. The null model assumed that the effective population size $\left(N_{\mathrm{e}}\right)$ of the black-faced spoonbill had been constant (constant size model). The alternative model assumed that the black-faced spoonbill had experienced a recent bottleneck event (recent bottleneck model) and therefore contained the following five parameters: $N$ e before the bottleneck, $N_{\mathrm{e}}$ for the bottleneck, $N_{\mathrm{e}}$ after bottleneck, the date of the initiation of the bottleneck and the date of the end of the bottleneck. We used a method implemented inFastsimcoal2 (Excoffier et al., 2013) to conduct model selection and parameter inference for the selected model. First, we generated the observed folded (minor allele) site frequency spectrum from the 215,722 unlinked autosomal SNPs of the black-faced spoonbill with the scripteasySFS (https://github.com/isaacovercast/easySFS\#easysfs). For the constant population size model, the prior for the $N$ e was set to 100-1,000 haploid individuals. Assuming a generation time of ten years and that the bottleneck event occurred between the 1950 and the 1980s(La Touche, 1931)'(Austin, 1948), the priors for the dates of bottleneck initiation ( $T$ bot $)$ and termination ( $T$ endbot) were set to 2-5 and 2-10 generations ago, respectively. Bracketing the long-term $N$ e $(1 / 2$ to $2 \times$ ) since the last glacial maximum estimated from the $S M C++$, we set the prior of $N$ e for the prebottleneck $(N$ anc $)$ to 7,500-30,000 haploid individuals. Considering black-faced spoonbill's population size of 288- 4000 since 1988, the priors for bottleneck $\left(N_{\text {bot }}\right)$ and post-bottleneck $N_{\mathrm{e}}\left(N_{\text {cur }}\right)$ were set to 2-100 and 100-1,000 haploid individuals respectively. Using uniform random samples from the priors of the two demographic models, we generated 100,000 folded SFSs for each model from the coalescent simulations and ran 40 optimization cycles to estimate each parameter and its composite likelihood in each simulation. The set of parameters with the highest likelihood was used for model selection. The simulation procedure described above was repeated 1,000 times, and a total of 5,000 sets of parameters were generated for each model. The parameters of the model from the set of simulations with the highest estimated likelihood was chosen as the best estimate of parameters for a given model. The Akaike information criterion (AIC) was used to compare the best simulation of the two demographic models to the observed folded site frequency spectrum (SFS). We used $\Delta$ AIC and AIC weight $(w)$ to evaluate which model better fitted the observed folded SFS. Then we ran the parameter estimation procedure under the best model to obtain 100 bootstrapped folded SFSs. Based on these bootstrapped folded SFS, we ran the parameter estimate procedure for each bootstrapped folded SFS again to compute the confidence interval of each parameter of the selected model.

\section{Genetic consequences of the recent bottleneck}

\section{Inbreeding}

In a small population random mating can result in a higher level of inbreeding. To assess the level of inbreeding, we used PLINK to calculate the number and length (>100 Kb) of ROHs through the autosomal genome for each black-faced and royal spoonbill individual.

We also calculated the genomic inbreeding coefficient, $F_{\mathrm{ROH}}$, which measures the proportion of ROHs in the autosomal genome(M. C. Keller et al., 2011). To calculate $F_{\text {ROH }}$, we divided the total length of ROH by the effective autosomal genome size (the length of non-N bases of the non-sex chromosomes).

In theory, a species with a higher inbreeding rate is expected to have a lower linkage disequilibrium (LD) decay rate(Slatkin, 2008). Therefore we also used PopLDdecay v. 3.40(Zhang, Dong, Xu, He, \& Yang, 2019) to calculate the decay of LD, $r^{2}$, for the two spoonbill species. To calculate the pair-wise ${ }^{2}$ between two SNPs, the maximum distance between them was set to $150 \mathrm{~Kb}$. 


\section{Genetic drift}

Genetic drift in a small population tends to cause the loss of rare alleles, such as singletons(Motoo Kimura \& Ohta, 1969). To evaluate the relative level of genetic drift in the black-faced spoonbill, we compared the folded site frequency spectrum of the unlinked autosomal SNPs between the two spoonbill species. We used the same folded SFS forFastSimcoal2 analysis for the black-faced spoonbill. For the royal spoonbill, we used the same filtering criteria as those for evaluating population structure to generate a set of 232,409 unlinked autosomal SNPs. Because the low frequency of rare alleles rendered by genetic drift could lead to a higher Tajima's $D$ value(Tajima, 1989), we also used VCFtools to calculate the Tajima's Dvalue for all autosomal SNPs of both spoonbill species.

\section{Relaxation of purifying selection}

Genetic drift can lead to relaxation of selection by lowering selection efficacy. To evaluate the level of selection relaxation, we used the descriptive model in RELAX (Wertheim et al., 2015) to infer a relaxation parameter $k$ for 7,835 orthologous genes between the two spoonbill species and an outgroup, the crested ibis Nipponia nippon (S. Li et al., 2014). A relaxation parameter $k>1$ indicates that the gene was under intensified selection, and $k<1$ implies relaxed selection. We counted the number of genes with a $k<1$ for each spoonbill species. First, we used the program Inparanoid v. 4.1(Remm, Storm, \& Sonnhammer, 2001) to identify homologous genes between the crested ibis and the black-faced spoonbill. Then, we usedgffread $\mathrm{v}$. 0.11.4 (https://github.com/gpertea/gffread) to extract the coding region sequences of each homologous gene from each black-faced and royal spoonbill individual. We used MACSEv0.9b1(Ranwez, Harispe, Delsuc, \& Douzery, 2011) to align the homologous sequences from all three species. We removed sequences that contained frameshifts and/or stop codons flagged by MACSE from the subsequent analysis. Then we used RAxMAL8 v. 8.2.12(Stamatakis, 2014) to draw the maximum likelihood gene tree of all 5,657 well-aligned homologous genes with the GTRGAMMA model. Gene trees of all orthologous genes from all three species in focus were used for the RELAX analysis. The likelihood ratio test in RELAX was used to estimatek for each homologous gene by comparing the model which fixed $k=1$ and the model which allowed $k$ to be estimated. The MRCA branch and each branch of each spoonbill species were alternatively set as the test branch in the RELAX analysis.

\section{Accumulation of deleterious mutations}

Relaxation of selection can result in an accumulation of deleterious mutations in the genome. To evaluate whether the genome of the black-faced spoonbill accumulated a significant number of deleterious mutations, we counted the number of nonsynonymous substitutions in both spoonbill species. First, we used ANNOVAR (Wang, Li, \& Hakonarson, 2010) to annotate substitutions in the coding region of both spoonbill species to two categories, synonymous and non-synonymous substitutions. Due to the lack of a suitable outgroup, we defined the dominant allele in a species as the ancestral allele, and the minor allele as the derived allele. Assuming that non-synonymous substitutions are deleterious, we counted the number of non-synonymous substitutions in each individual as accumulated deleterious mutations also known as genetic load. To further quantify the deleterious effect of non-synonymous substitutions, we used the Grantham score(Grantham, 1974) to measure their potential phenotypic effect. The Grantham score quantifies the differences in physiochemical similarity and evolutionary exchangeability between two amino acids, where a higher score indicates more physiochemical difference and lower evolutionary exchangeability (i.e. higher deleterious phenotypic effect of nonsynonymous substitution). Grantham scores can be classified into three categories(Chun \& Fay, 2009), conservative changes (0-50), moderate changes (51-100) and radical changes (>100). Assuming conservative changes in amino acids to be neutral and different deleterious non-synonymous substitutions to have an independent and additive effect, we counted the accumulated deleterious effect of non-synonymous substitutions by summing up the Grantham scores categorized as moderate or radical changes for each individual.

\section{References}


Alexander, D. H., \& Lange, K. (2011). Enhancements to the ADMIXTURE algorithm for individual ancestry estimation. BMC Bioinformatics ,12 (1), 246. doi: 10.1186/1471-2105-12-246

Allendorf, F. W. (1986). Genetic drift and the loss of alleles versus heterozygosity. Zoo Biology , 5 (2), 181-190. doi: 10.1002/zoo.1430050212

Austin, O. L. Jr. (1948). The birds of Korea. Bull. Mus. Comp. Zool. , 101 , 1-301.

Axelsson, E., Smith, N. G. C., Sundström, H., Berlin, S., \& Ellegren, H. (2004). Male-Biased Mutation Rate and Divergence in Autosomal, Z-Linked and W-Linked Introns of Chicken and Turkey. Molecular Biology and Evolution, 21 (8), 1538-1547. doi: 10.1093/molbev/msh157

Baillie, J., Hilton-Taylor, C., \& Stuart, S. N. (2004). 2004 IUCN Red List of threatened species: A global species assessment. IUCN.

Barrett, R. D. H., \& Schluter, D. (2008). Adaptation from standing genetic variation. Trends in Ecology $\&$ Evolution , 23 (1), 38-44. doi: 10.1016/j.tree.2007.09.008

Bijlsma, R., \& Loeschcke, V. (2012). Genetic erosion impedes adaptive responses to stressful environments. Evolutionary Applications ,5 (2), 117-129. doi: 10.1111/j.1752-4571.2011.00214.x

BirdLife International. (2017). Platalea minor. In The IUCN Red List of Threatened Species 2017: E.T22697568A119347801 . IUCN. Retrieved from http://dx.doi.org/10.2305/IUCN.UK.20173.RLTS.T22697568A119347801.en.

BirdLife International 2000. (2000). Platalea minor. In The IUCN Red List of Threatened Species 2000: E.T22697568A33426376. IUCN.

Bolger, A. M., Lohse, M., \& Usadel, B. (2014). Trimmomatic: A flexible trimmer for Illumina sequence data. Bioinformatics ,30 (15), 2114-2120. doi: 10.1093/bioinformatics/btu170

Bowkett, A. E. (2009). Recent Captive-Breeding Proposals and the Return of the Ark Concept to Global Species Conservation. Conservation Biology , 23 (3), 773-776. doi: 10.1111/j.1523-1739.2008.01157.x

Brondizio, E. S., Settele, J., Díaz, S., \& Ngo, H. T. (Series Eds.). (2019). IPBES (2019): Global assessment report on biodiversity and ecosystem services of the Intergovernmental Science-Policy Platform on Biodiversity and Ecosystem Services (p. 56). Bonn, Germany: IPBES secetariat.

Charlesworth, D., \& Charlesworth, B. (1987). Inbreeding depression and its evolutionary consequences. Annual Review of Ecology and Systematics , 18 (1), 237-268.

Chesser, R. T., Yeung, C. K. L., Yao, C. T., Tian, X. H., \& Li, S.-H. (2010). Molecular phylogeny of the spoonbills (Aves: Threskiornithidae) based on mitochondrial DNA. Zootaxa , (2603), 53-60.

Cho, Y. S., Hu, L., Hou, H., Lee, H., Xu, J., Kwon, S., . . Bhak, J. (2013). The tiger genome and comparative analysis with lion and snow leopard genomes. Nature Communications , 4 , 2433. doi: 10.1038/ncomms3433

Chong, J.-R., Pak, U.-I., Rim, C.-Y., \& Kim, T.-S. (1996). Breeding biology of black-faced spoonbill Platalea minor . Strix ,14, 1-10.

Chun, S., \& Fay, J. C. (2009). Identification of deleterious mutations within three human genomes. Genome Research , 19 (9), 1553-1561. doi: 10.1101/gr.092619.109

Cooke, A. S. (1973). Shell thinning in avian eggs by environmental pollutants. Environmental Pollution (1970) , 4 (2), 85-152. doi: 10.1016/0013-9327(73)90009-8

Crow, J. F., \& Kimura, M. (1970). An introduction to population genetics theory. New York: Harper \& Row, Publishers. Retrieved from https://www.cabdirect.org/cabdirect/abstract/19710105376

Danecek, P., Auton, A., Abecasis, G., Albers, C. A., Banks, E., DePristo, M. A., .. Durbin, R. (2011). The variant call format and VCFtools. Bioinformatics , 27 (15), 2156-2158. doi: 10.1093/bioinformatics/btr330 
Ding, W., Lei, F., Yin, Z., \& Liu, R. (1999). The breeding sites of the black-faced spoonbill Platalea minor have been discovered in Northern China. Bird Conservation International , 9 , 284.

Do, R., Balick, D., Li, H., Adzhubei, I., Sunyaev, S., \& Reich, D. (2015). No evidence that selection has been less effective at removing deleterious mutations in Europeans than in Africans. Nature Genetics , 47 (2), 126-131. doi: 10.1038/ng.3186

Dobson, A. P., Bradshaw, A. D., \& Baker, A. J. M. (1997). Hopes for the Future: Restoration Ecology and Conservation Biology. Science, 277 (5325), 515-522. doi: 10.1126/science.277.5325.515

Ewen, J. G., Walker, L., Canessa, S., \& Groombridge, J. J. (2015). Improving supplementary feeding in species conservation. Conservation Biology , 29 (2), 341-349. doi: 10.1111/cobi.12410

Excoffier, L., Dupanloup, I., Huerta-Sánchez, E., Sousa, V. C., \& Foll, M. (2013). Robust demographic inference from genomic and SNP data.PLoS Genetics , 9 (10), e1003905-e1003905. doi: 10.1371/journal.pgen.1003905

Faber, R. A., \& Hickey, J. J. (1973). Eggshell thinning, chlorinated hydrocarbons, and mercury in inland aquatic bird egg, 1969 and 1970.Pesticide Monitoring , 7 , 27-36.

Frankham, R. (1995). Effective population size/adult population size ratios in wildlife: A review. Genetics Research , 66 (2), 95-107. doi: 10.1017/S0016672300034455

Frankham, R., Bradshaw, C. J. A., \& Brook, B. W. (2014). Genetics in conservation management: Revised recommendations for the 50/500 rules, Red List criteria and population viability analyses. Biological Conservation , 170 , 56-63. doi: 10.1016/j.biocon.2013.12.036

Fu, W., Gittelman, R. M., Bamshad, M. J., \& Akey, J. M. (2014). Characteristics of Neutral and Deleterious Protein-Coding Variation among Individuals and Populations. The American Journal of Human Genetics, 95 (4), 421-436. doi: 10.1016/j.ajhg.2014.09.006

Gattepaille, L. M., Jakobsson, M., \& Blum, M. G. (2013). Inferring population size changes with sequence and SNP data: Lessons from human bottlenecks. Heredity , 110 (5), 409-419. doi: 10.1038/hdy.2012.120

Gemmell, N. J., \& Akiyama, S. (1996). An efficient method for the extraction of DNA from vertebrate tissues. Trends in Genetics: TIG , 12 (9), 338-339. doi: 10.1016/s0168-9525(96)80005-9

Gnerre, S., MacCallum, I., Przybylski, D., Ribeiro, F. J., Burton, J. N., Walker, B. J., .. Jaffe, D. B. (2011). High-quality draft assemblies of mammalian genomes from massively parallel sequence data.Proceedings of the National Academy of Sciences , 108 (4), 1513-1518. doi: 10.1073/pnas.1017351108

Gómez-Sanchez, D., Olalde, I., Sastre, N., Ensenat, C., Carrasco, R., Marques-Bonet, T., .. Ramirez, O. (2018). On the path to extinction: Inbreeding and admixture in a declining grey wolf population. Molecular Ecology , 27 (18), 3599-3612. doi: 10.1111/mec.14824

Grabherr, M. G., Haas, B. J., Yassour, M., Levin, J. Z., Thompson, D. A., Amit, I., .. Regev, A. (2011). Trinity: Reconstructing a full-length transcriptome without a genome from RNA-Seq data.Nature Biotechnology , 29 (7), 644-652. doi: 10.1038/nbt.1883

Grabherr, M. G., Russell, P., Meyer, M., Mauceli, E., Alfoldi, J., Di Palma, F., \& Lindblad-Toh, K. (2010). Genome-wide synteny through highly sensitive sequence alignment: Satsuma. Bioinformatics ,26 (9), 11451151. doi: 10.1093/bioinformatics/btq102

Grantham, R. (1974). Amino acid difference formula to help explain protein evolution. Science, 185 (4154), 862-864. Scopus. doi: 10.1126/science.185.4154.862

Grier, J. W. (1982). Ban of DDT and subsequent recovery of Reproduction in bald eagles. Science, 218 (4578), 1232-1235. doi: 10.1126/science.7146905 
Grossen, C., Guillaume, F., Keller, L. F., \& Croll, D. (2020). Purging of highly deleterious mutations through severe bottlenecks in Alpine ibex. Nature Communications , 11 (1), 1-12. doi: 10.1038/s41467-020-14803-1

Haas, B. J., Delcher, A. L., Mount, S. M., Wortman, J. R., Smith, R. K., Hannick, L. I., .. . White, O. (2003). Improving the Arabidopsis genome annotation using maximal transcript alignment assemblies.Nucleic Acids Research , 31 (19), 5654-5666. doi: 10.1093/nar/gkg770

Hermisson, J., \& Pennings, P. S. (2005). Soft Sweeps: Molecular Population Genetics of Adaptation From Standing Genetic Variation.Genetics , 169 (4), 2335-2352. doi: 10.1534/genetics.104.036947

Hill, W. G., \& Robertson, A. (1966). The effect of linkage on limits to artificial selection. Genetics Research , 8 (3), 269-294. doi: 10.1017/S0016672300010156

Jones, F. C., Grabherr, M. G., Chan, Y. F., Russell, P., Mauceli, E., Johnson, J., ... Kingsley, D. M. (2012). The genomic basis of adaptive evolution in threespine sticklebacks. Nature ,484 (7392), 55-61. doi: $10.1038 /$ nature10944

Keller, L. F., \& Waller, D. M. (2002). Inbreeding effects in wild populations. Trends in Ecology \& Evolution , 17 (5), 230-241. doi: 10.1016/S0169-5347(02)02489-8

Keller, M. C., Visscher, P. M., \& Goddard, M. E. (2011). Quantification of Inbreeding Due to Distant Ancestors and Its Detection Using Dense Single Nucleotide Polymorphism Data. Genetics , 189 (1), 237249. doi: 10.1534 /genetics.111.130922

Kennerley, K. J. (1990). A review of the status and distribution of black-faced spoonbill. In V. Pickens (Ed.), The Hong Kong bird report 1989 . Hong Kong: Hong Kong Bird Watching Society.

Kimura, M. (1962). On the probability of fixation of mutant genes in a population. Genetics , 47, 713-719.

Kimura, Motoo, Maruyama, T., \& Crow, J. F. (1963). The Mutation Load in Small Populations. Genetics , $48(10), 1303-1312$.

Kimura, Motoo, \& Ohta, T. (1969). The Average Number of Generations until Fixation of a Mutant Gene in a Finite Population. Genetics ,61 (3), 763-771.

King, K. A., Meeker, D. L., \& Swineford, D. M. (1980). White-faced ibis populations and pollutants in Texas, 1969-1976. Southwestern Naturalist , 25 , 225-240.

Kirkpatrick, M., \& Jarne, P. (2000). The Effects of a Bottleneck on Inbreeding Depression and the Genetic Load. The American Naturalist , 155 (2), 154-167. doi: 10.1086/303312

La Touche, J. (1931). A handbook of birds of eastern China . London: Taylor \& Francis.

Lai, Y.-T., Yeung, C. K. L., Omland, K. E., Pang, E.-L., Hao, Y., Liao, B.-Y., .. L Li, S.-H. (2019). Standing genetic variation as the predominant source for adaptation of a songbird. Proceedings of the National Academy of Sciences , 116 (6), 2152-2157. doi: 10.1073/pnas.1813597116

Lande, R., \& Shannon, S. (1996). The role of genetic variation in adaptation and population persistence in a changing environment.Evolution; International Journal of Organic Evolution ,50 (1), 434-437. doi: $10.2307 / 2410812$

Larson, C. (2015). Hostile shores. Science , 350 (6257), 150-152. doi: 10.1126/science.350.6257.150

Li, H. (2011). A statistical framework for SNP calling, mutation discovery, association mapping and population genetical parameter estimation from sequencing data. Bioinformatics , 27 (21), 2987-2993. doi: 10.1093/bioinformatics/btr509

Li, H., \& Durbin, R. (2010). Fast and accurate long-read alignment with Burrows-Wheeler transform. Bioinformatics , 26 (5), 589-595. doi: 10.1093/bioinformatics/btp698 
Li, S., Li, B., Cheng, C., Xiong, Z., Liu, Q., Lai, J., .. Yan, J. (2014). Genomic signatures of near-extinction and rebirth of the crested ibis and other endangered bird species. Genome Biology ,15 (12), 557-557. doi: 10.1186/s13059-014-0557-1

Litvinenko, N. M., \& Shibaev, Y. V. (2005). Black-faced SpoonbillPlatalea minor. In Red Data Book Primorsky krai. Animals.(p. 209). Vladivostok.

Lohmueller, K. E. (2014). The Impact of Population Demography and Selection on the Genetic Architecture of Complex Traits. PLOS Genetics , 10 (5), e1004379. doi: 10.1371/journal.pgen.1004379

Ma, Z., Melville, D. S., Liu, J., Chen, Y., Yang, H., Ren, W., ... Li, B. (2014). Rethinking China's new great wall. Science ,346 (6212), 912-914. doi: 10.1126/science.1257258

Martin, G., \& Lenormand, T. (2006). The Fitness Effect of Mutations Across Environments: A Survey in Light of Fitness Landscape Models.Evolution , 60 (12), 2413-2427. doi: 10.1111/j.0014-3820.2006.tb01878.x

Maruyama, T., \& Fuerst, P. A. (1985). Population bottlenecks and nonequilibrium models in population genetics. II. Number of alleles in a small population that was formed by a recent bottleneck.Genetics , 111 (3), 675-689.

Mason, N. A., Pulgarin, P., Cadena, C. D., \& Lovette, I. J. (2020). De Novo assembly of a high-quality reference genome for the horned lark (Eremophila alpestris ). G3 Genes|Genomes|Genetics, 10 (2), 475-478. doi: $10.1534 / \mathrm{g} 3.119 .400846$

Matheu, E., \& del Hoyo, J. (1992). Family Threskiornithidae (Ibises and Spoonbills). In Handbook of the birds of theworld (Vol. 1, pp. 472-507). Barcelona: Lynx Edicions.

Mays, H. L., Hung, C.-M., Shaner, P.-J., Denvir, J., Justice, M., Yang, S.-F., .. Primerano, D. A. (2018). Genomic Analysis of Demographic History and Ecological Niche Modeling in the Endangered Sumatran Rhinoceros Dicerorhinus sumatrensis. Current Biology ,28 (1), 70-76.e4. doi: 10.1016/j.cub.2017.11.021

McQuillan, R., Leutenegger, A.-L., Abdel-Rahman, R., Franklin, C. S., Pericic, M., Barac-Lauc, L., ... Wilson, J. F. (2008). Runs of Homozygosity in European Populations. The American Journal of Human Genetics , 83 (3), 359-372. doi: 10.1016/j.ajhg.2008.08.007

Newton, I., \& Wyllie, I. (1992). Recovery of a Sparrowhawk Population in Relation to Declining Pesticide Contamination. Journal of Applied Ecology , 29 (2), 476-484. JSTOR. doi: 10.2307/2404515

Pimm, S. L., Jenkins, C. N., Abell, R., Brooks, T. M., Gittleman, J. L., Joppa, L. N., .. Sexton, J. O. (2014). The biodiversity of species and their rates of extinction, distribution, and protection.Science, 344 (6187). doi: 10.1126/science.1246752

Poole, A. F. (1989). Ospreys: A natural and unnatural history . Cambridge: Cambridge University Press.

Purcell, S., Neale, B., Todd-Brown, K., Thomas, L., Ferreira, M. A. R., Bender, D., .. Sham, P. C. (2007). PLINK: A Tool Set for Whole-Genome Association and Population-Based Linkage Analyses. The American Journal of Human Genetics , 81 (3), 559-575. doi: 10.1086/519795

Ranwez, V., Harispe, S., Delsuc, F., \& Douzery, E. J. P. (2011). MACSE: Multiple Alignment of Coding SEquences Accounting for Frameshifts and Stop Codons. PLOS ONE , 6 (9), e22594. doi: 10.1371/journal.pone.0022594

Remm, M., Storm, C. E. V., \& Sonnhammer, E. L. L. (2001). Automatic clustering of orthologs and in-paralogs from pairwise species comparisons. Journal of Molecular Biology , 314 (5), 1041-1052. doi: 10.1006/jmbi.2000.5197

Ridgway, J., \& Shimmield, G. (2002). Estuaries as Repositories of Historical Contamination and their Impact on Shelf Seas.Estuarine, Coastal and Shelf Science, 55 (6), 903-928. doi: 10.1006/ecss.2002.1035 
Risebrough, R. W. (1986). Pesticides and Bird Populations. In R. Johnston (Ed.), Current Ornithology (Vol. 3, pp. 397-427). Springer.

Robertson, A., \& Waddington, C. H. (1960). A theory of limits in artificial selection. Proceedings of the Royal Society of London. Series B. Biological Sciences , 153 (951), 234-249. doi: 10.1098/rspb.1960.0099

Robinson, J. A., Ortega-Del Vecchyo, D., Fan, Z., Kim, B. Y., vonHoldt, B. M., Marsden, C. D., .. Wayne, R. K. (2016). Genomic Flatlining in the Endangered Island Fox. Current Biology , 26 (9), 1183-1189. doi: 10.1016/j.cub.2016.02.062

Rubenstein, D. R., Chamberlain, C. P., Holmes, R. T., Ayres, M. P., Waldbauer, J. R., Graves, G. R., \& Tuross, N. C. (2002). Linking Breeding and Wintering Ranges of a Migratory Songbird Using Stable Isotopes. Science , 295 (5557), 1062-1065. doi: 10.1126/science.1067124

Shibaev, Y. V. (2010). Breeding of the Black-faced Spoonbill (Platalea minor ) in Peter the Great Bay (Primorye, Russia). Situation and prospects. In Annual Report of Pro Natura Fund(Vol. 19, pp. 151-164).

Simons, Y. B., Turchin, M. C., Pritchard, J. K., \& Sella, G. (2014). The deleterious mutation load is insensitive to recent population history. Nature Genetics , 46 (3), 220-224. doi: 10.1038/ng.2896

Slatkin, M. (2008). Linkage disequilibrium-Understanding the evolutionary past and mapping the medical future. Nature Reviews Genetics , 9 (6), 477-485. doi: 10.1038/nrg2361

Smith, R. K., Pullin, A. S., Stewart, G. B., \& Sutherland, W. J. (2010). Effectiveness of Predator Removal for Enhancing Bird Populations. Conservation Biology , 24 (3), 820-829. doi: 10.1111/j.1523-1739.2009.01421.x

Stamatakis, A. (2014). RAxML version 8: A tool for phylogenetic analysis and post-analysis of large phylogenies. Bioinformatics ,30 (9), 1312-1313. doi: 10.1093/bioinformatics/btu033

Stanke, M., \& Morgenstern, B. (2005). AUGUSTUS: A web server for gene prediction in eukaryotes that allows user-defined constraints.Nucleic Acids Research, 33 (Web Server issue), W465-W467. doi: 10.1093/nar/gki458

Sung, Y.-H., Tse, I. W.-L., \& Yu, Y.-T. (2018). Population trends of the Black-faced Spoonbill Platalea minor : Analysis of data from international synchronised censuses. Bird Conservation International , 28 (1), 157-167. doi: 10.1017/S0959270917000016

Szafraniec, K., Borts, R. H., \& Korona, R. (2001). Environmental stress and mutational load in diploid strains of the yeast Saccharomyces cerevisiae. Proceedings of the National Academy of Sciences of the United States of America, 98 (3), 1107-1112.

Tajima, F. (1989). The effect of change in population size on DNA polymorphism. Genetics , 123 (3), $597-601$.

Terhorst, J., Kamm, J. A., \& Song, Y. S. (2017). Robust and scalable inference of population history from hundreds of unphased whole genomes.Nature Genetics , 49 (2), 303-309. doi: 10.1038/ng.3748

Ueta, M., Melville, D. S., Wang, Y., Ozaki, K., Kanai, Y., Leader, P. J., .. Kuo, C.-Y. (2002). Discovery of the breeding sites and migration routes of Black-faced Spoonbills Platalea minor.Ibis , 144 (2), 340-343. doi: 10.1046/j.1474-919X.2002.00037.x

Wang, K., Li, M., \& Hakonarson, H. (2010). ANNOVAR: Functional annotation of genetic variants from high-throughput sequencing data.Nucleic Acids Research , 38 (16), e164-e164. doi: 10.1093/nar/gkq603

Warren, W. C., Hillier, L. W., Tomlinson, C., Minx, P., Kremitzki, M., Graves, T., .. Cheng, H. H. (2017). A New Chicken Genome Assembly Provides Insight into Avian Genome Structure. G3: Genes, Genomes, Genetics , 7 (1), 109-117. doi: 10.1534/g3.116.035923 
Waterhouse, R. M., Seppey, M., Simao, F. A., Manni, M., Ioannidis, P., Klioutchnikov, G., ... Zdobnov, E. M. (2018). BUSCO Applications from Quality Assessments to Gene Prediction and Phylogenomics.Molecular Biology and Evolution , 35 (3), 543-548. doi: 10.1093/molbev/msx319

Weber, K. (2004). Population Size and Long-term Selection. In J. Janick (Series Ed.), Plant Breeding Reviews, Part1: Long-term Selection: Maize (Vol. 24, pp. 249-268). Hoboken, New Jersey: John Wiley \& Sons.

Wertheim, J. O., Murrell, B., Smith, M. D., Kosakovsky Pond, S. L., \& Scheffler, K. (2015). RELAX: Detecting Relaxed Selection in a Phylogenetic Framework. Molecular Biology and Evolution ,32 (3), 820832. doi: $10.1093 / \mathrm{molbev} / \mathrm{msu} 400$

Wong, M. H., Leung, A. O. W., Chan, J. K. Y., \& Choi, M. P. K. (2005). A review on the usage of POP pesticides in China, with emphasis on DDT loadings in human milk. Chemosphere, 60 (6), 740-752. doi: 10.1016/j.chemosphere.2005.04.028

Yang, J., Lee, S. H., Goddard, M. E., \& Visscher, P. M. (2011). GCTA: A Tool for Genome-wide Complex Trait Analysis. American Journal of Human Genetics , 88 (1), 76-82. doi: 10.1016/j.ajhg.2010.11.011

Yeung, C. K. L., Tsai, P.-W., Chesser, R. T., Lin, R.-C., Yao, C.-T., Tian, X.-H., \& Li, S.-H. (2011). Testing Founder Effect Speciation: Divergence Population Genetics of the Spoonbills Platalea regiaand Pl. minor (Threskiornithidae, Aves). Molecular Biology and Evolution , 28 (1), 473-482. doi: 10.1093/molbev/msq210

Yeung, C. K.-L., Yao, C.-T., Hsu, Y.-C., Wang, J.-P., \& Li, S.-H. (2006). Assessment of the historical population size of an endangered bird, the black-faced spoonbill (Platalea minor ) by analysis of mitochondrial DNA diversity. Animal Conservation , 9 (1), 1-10. doi: 10.1111/j.1469-1795.2005.00007.x

Yu, Y. T., Li, C. H., Tse, I. W. L., \& Fong, H. H. N. (2020). The International Black-faced Spoonbill Census 2020 . Hong Kong: Black-faced Spoonbill Research Group, The Hong Kong Bird Watching Society.

Zhang, C., Dong, S.-S., Xu, J.-Y., He, W.-M., \& Yang, T.-L. (2019). PopLDdecay: A fast and effective tool for linkage disequilibrium decay analysis based on variant call format files. Bioinformatics (Oxford, England) , 35 (10), 1786-1788. doi: 10.1093/bioinformatics/bty875

Zhao, S., Zheng, P., Dong, S., Zhan, X., Wu, Q., Guo, X., ... Wei, F. (2013). Whole-genome sequencing of giant pandas provides insights into demographic history and local adaptation. Nature Genetics , 45 (1), 67-71. doi: $10.1038 / \mathrm{ng} .2494$

Data Accessibility:

DNA sequences: NCBI BioProject ID: PRJNA597661

\section{Figure legends}

Figure 1. A. Distribution map of the black-faced (red: breeding range; gold: wintering range) and royal spoonbills (blue; modified from Hancock et al. 1992). The arrows indicate the two sampling sites, Tainan, Taiwan (blue) and Hong Kong (orange), of the black-faced spoonbill used in current study. B. The crossvalidation (CV) error of the ADMIXTURE analysis $(K: 1-5)$ of the black-faced spoonbill from Taiwan and Hong Kong was smallest when $K$ was equal to 1 . The origin of samples is labeled at the bottom: T: Tainan, Taiwan; HK: Hong Kong. C. Samples of the black-faced spoonbill from Tainan, Taiwan and Hong Kong did not form distinct clusters in the plot of scores of PC1 (1.33\% of genetic variance) versus PC2 (1.28\% of genetic variance). The origin of the samples is indicated by colors: blue: Tainan, Taiwan; orange: Hong Kong.

Figure 2. A. The historical effective population size $\left(N_{\text {e }}\right)$ trajectories estimated by $S M C++$ for both the black-faced and the royal spoonbill since the last glacial maximum (c.a. 22,000 years before the present); the full $N$ e trajectories of the two spoonbill species estimated by $S M C++$ are shown within the inset. The black-faced spoonbill in magenta and the royal spoonbill in apricot. B. Demographic parameters of the 
recent bottleneck model for the black-faced spoonbill estimated from simulations of Fastsimcoal2 based on the folded site frequency spectrum of the 215,722 unlinked autosomal SNPs. The solid line is the maximum likelihood estimate of various $N_{\mathrm{e}}$; The dashed line is the $95 \% \mathrm{CI}$ of $N$ anc and $N$ cur. $T$ bot: initiation time of the bottleneck; $T$ endbot: termination time of the bottleneck; $N$ anc: long term $N$ e before the bottleneck; $N$

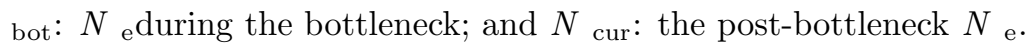

Figure 3. A. The black-faced spoonbills sequenced had more runs of homozygosity (> $100 \mathrm{~Kb}$; NROH) and longer total runs of homozygosity ( $\mathrm{SROH}$ ) than the royal spoonbills sequenced. The black-faced spoonbills are shown in circles, and the royal spoonbills in triangles. B. The linkage $\left(r^{2}\right)$ between SNPs on the same scaffold decayed more slowly in the black-faced spoonbill genome than in the royal spoonbill genome. C. The proportion of singletons in the folded site frequency spectrum for unlinked $\left(r^{2}<0.2\right)$ autosomal SNPs was significantly lower in the black-faced spoonbill genomes (21.9\%) than in the royal spoonbill genome $(31.7 \%$; $\left.\chi^{2}=24.82, p=0.000\right)$. D. The Tajima's $D$ value for all autosomal SNPs of the black-faced spoonbill $(0.24 \pm$ $0.98, n=4,522,032)$ is higher than that of the royal spoonbill $(0.05 \pm 0.99, n=4,437,044 ; t=296.51, p<$ 0.001). The black-faced spoonbill is shown in magenta and the royal spoonbill in apricot.

Figure 4. A. Significantly more genes in the black-faced spoonbill $(2,094,37.0 \%)$ are under relaxed selection (relaxation parameter $k<1, p<0.05)$ than in the royal spoonbill $\left(1,542,27.2 \% ; \chi^{2}=123.04, p=2.2 \mathrm{e}-16\right.$; Figure 4A); but not for genes under intensified selection $\left.\chi^{2}=3.17, p=0.075\right)$. B. The mean number of nonsynonymous substitutions found in each individual of the black-faced spoonbill genome (8294.00土332.66 substitutions) is higher than that found in the royal spoonbill genome (8025.78 \pm 211.77 substitutions; $t$ $=-1.91, p<0.035)$. C. The average Grantham's score for each deleterious nonsynonymous substitution (Grantham's score $>50$ ) is significantly higher for the black-faced spoonbill (56.98 \pm 0.69$)$ than for the royal spoonbill ( $53.25 \pm 1.83$; two-sample $t$ test $=6.5154, p=3.6 \mathrm{e}-6)$. D. The ratio of homozygous to heterozygous nonsynonymous substitutions in the genome of the black-faced spoonbill $(0.13 \pm 0.04)$ is higher than that in the royal spoonbill genome $(0.11 \pm 0.007 ; t=-1.86, p<0.04)$. The black-faced spoonbill is shown in magenta and the royal spoonbill in apricot.
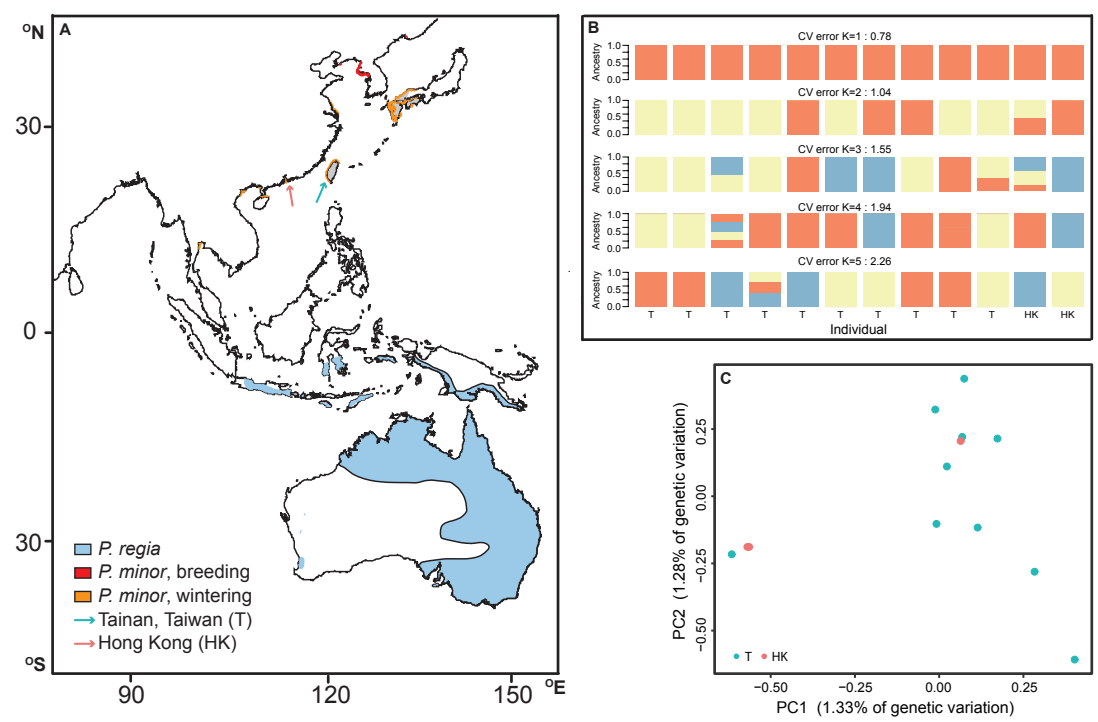

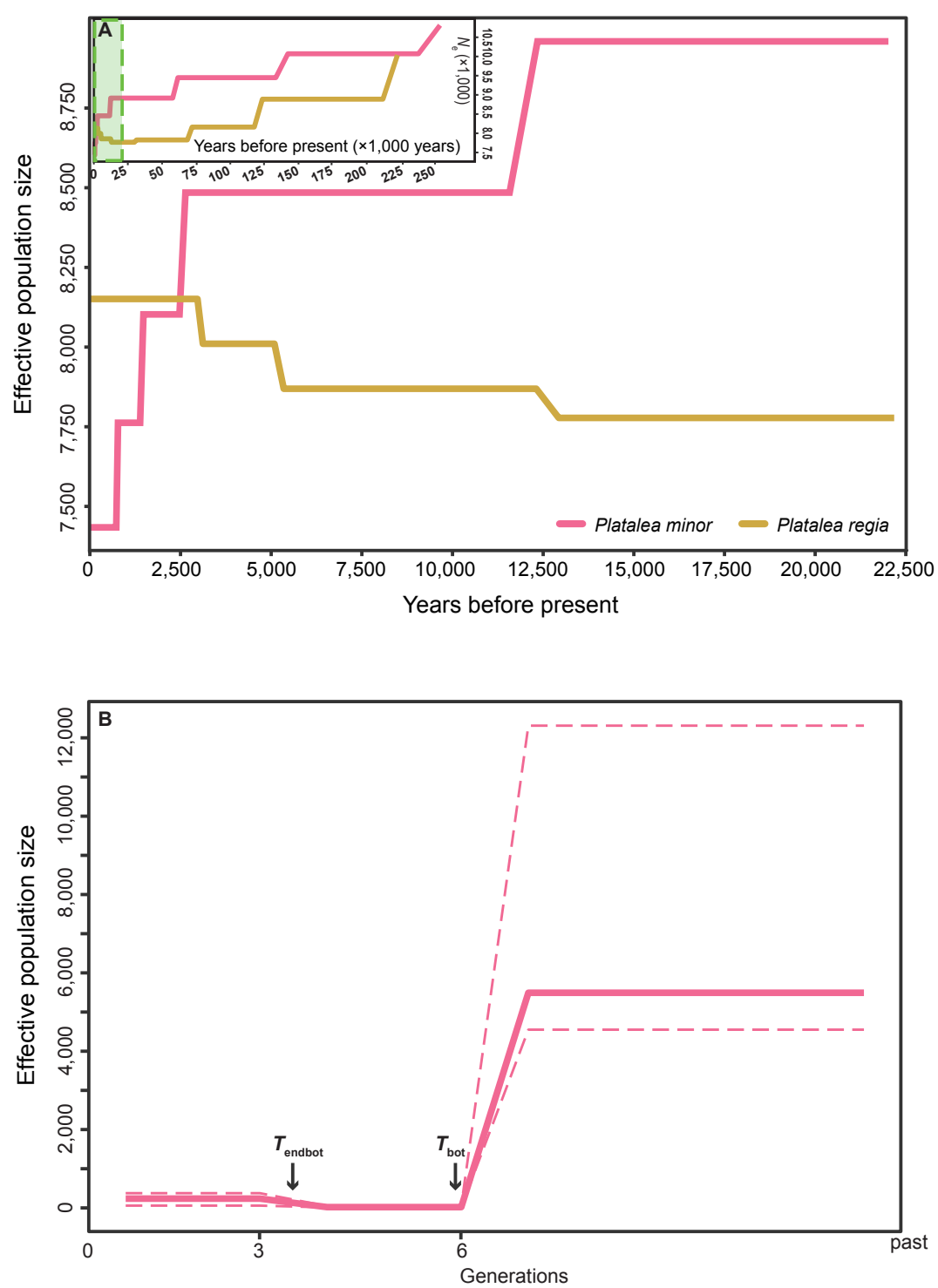

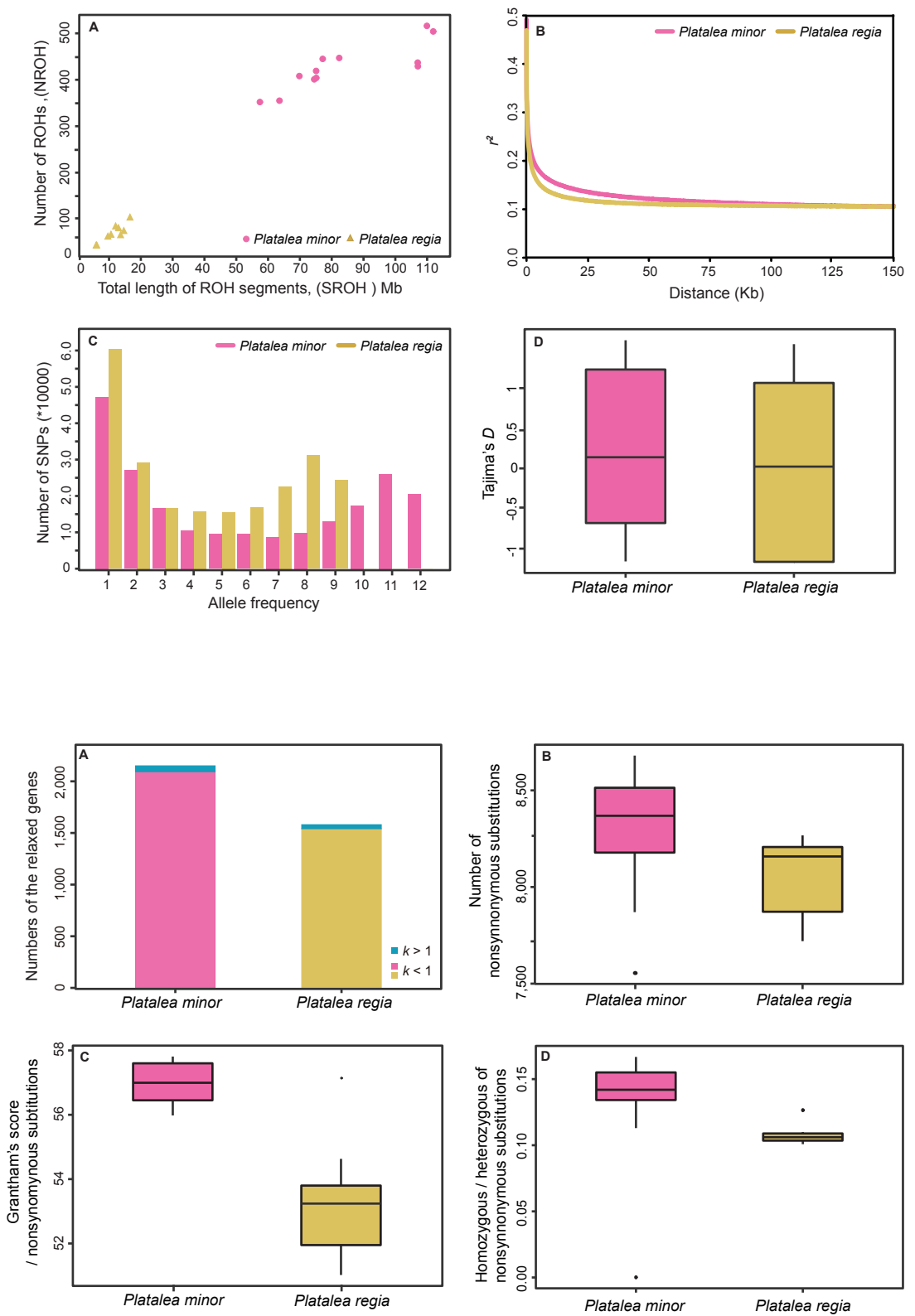